\title{
Determining the optimum length of a bridge opening with a specified reliability level of water runoff
}

\author{
Sergey Evdokimov ${ }^{1}$, and Tatiana Dormidontova ${ }^{1, *}$ \\ ${ }^{1}$ Samara State Technical University, Institute of Architecture and Civil Engineering, \\ 194, Molodogvardeyskaya St., 443001, Samara, Russia
}

\begin{abstract}
Current trends in construction are aimed at providing reliability and safety of engineering facilities. According to the latest government regulations for construction, the scientific approach to engineering research, design, construction and operation of construction projects is a key priority. The reliability of a road depends on a great number of factors and characteristics of their statistical compounds (sequential and parallel). A part of a road with such man-made structures as a bridge or a pipe is considered as a system with a sequential element connection. The overall reliability is the multiplication of the reliability of these elements. The parameters of engineering structures defined by analytical dependences are highly volatile because of the inaccuracy of the defining factors. However each physical parameter is statistically unstable that is evaluated by variable coefficient of their values. It causes the fluctuation in the parameters of engineering structures. Their study may result in the changes in general and particular design rules in order to increase the reliability. The paper gives the grounds for these changes by the example of a bridge. It allows calculating its optimum length with a specified reliability level of water runoff under the bridge.
\end{abstract}

\section{Introduction}

At present, safety and reliability of construction engineering facilities on roads is a matter of great concern. The paper reports on the reliability of a road that includes the reliability of its elements- road surfacing, geometric elements, man-made structures [1-7].

The reliability is closely related to the probability of failures or risks. The reliability of an auto-road and man-made structures on it is much less important, less durable and has absolute serviceability.

The low reliability of a road depends on a large number of factors and characteristics of their statistical sequential and parallel compounds. A part of a road with such man-made structures as a bridge or a pipe is considered as a system with a sequential connection of elements. The overall reliability is the multiplication of the reliability of these elements. In this regard, the methodology proposed in this research work aimed at determining the

\footnotetext{
* Corresponding author: adisk63@yandex.ru
} 
optimum length of the bridge opening with a specified reliability level of water runoff seems to be relevant.

\section{Materials and Methods}

In order to provide the reliability of slopes stabilized with soil, its gradient $m$ should correspond to the calculated shift angle $\psi$, Formula (1):

$$
\operatorname{tg} \psi=\frac{1}{\mathrm{~K}_{3}}\left(\operatorname{tg} \varphi+\frac{c}{p_{h}}\right),
$$

where, $K_{3}$ - assurance coefficient $\left(K_{3}=1\right.$, as it corresponds to the limit state); $\varphi$ - angle of internal friction; $c$ - adhesion;

$$
m=\frac{1}{\operatorname{tg} \varphi+\frac{c}{p_{h}}}
$$

$p_{h}$ - pressure on ground: $p_{h}=\cos ^{2} \varphi \cdot h \cdot \gamma \cdot 1 \mathrm{rm} ; \cos ^{2} \varphi \cdot h$ - ground area on the cross-section of a slope; $\gamma$ - soil density; $1 \mathrm{rm}$ - running meter. (2)

Let's find the derivatives of the function $\mathbf{m}$ from $\gamma, \boldsymbol{\varphi}$, and $\boldsymbol{c}$ calculated by the formula

$$
\begin{gathered}
\frac{\partial m}{\partial \varphi}=\frac{-1}{\operatorname{tg} \varphi^{2}+\frac{c}{p_{h}}} \\
\frac{\partial m}{\partial c}=\frac{-1}{\operatorname{tg} \varphi+\frac{c^{2}}{p_{h}}} \\
\frac{\partial m}{\partial \gamma}=\frac{-1}{\operatorname{tg} \varphi+\frac{c}{F \cdot \gamma^{2}}}
\end{gathered}
$$

To determine the reliability of a slope the authors use the existing normative values for the gradient.

It should be noted, the reliability is calculated as follows, Formula (3):

$$
P=f(\mathrm{Z})=f\left(\frac{\bar{m}-m_{\text {norm }}}{\sqrt{\sigma_{m}^{2}+\sigma_{\text {norm }}^{2}}}\right)
$$

To determine the reliability of superelevation Formula (4) should be used.

$$
i_{\text {superel }}=V^{2} / g R-M=v^{2} / g R-C_{I I}
$$

Where: $V$ - car speed in superelevation; $\mathrm{g}$ - acceleration of gravity $\left(\mathrm{g}=9,81 \mathrm{~m} / \mathrm{s}^{2}\right) ; R-$ turning radius $\left(R_{\min }=800 \mathrm{~m}\right.$ for a road of Category II); $M$ - sideway force coefficient; $C_{I I}-$ coefficient of adhesion of car wheels with the ground.

Then standard for the superelevation gradient should be calculated by (5). 


$$
\sigma_{i}=\sqrt{\left(2 \cdot \frac{\bar{V}}{g \cdot R} \cdot \sigma_{V}\right)^{2}+1 \cdot \sigma_{\mu}{ }^{2}}
$$

Then a bridge capability is calculated. To determine the probability of exceeding the flow through the specified bridge section the following formula should be used (6):

$$
Q_{C}=1.35 H^{2 / 3} \cdot b
$$

Where, $H=1,45 V_{C} / g$ - head of water; $V_{C}$ - flow velocity under the bridge; $g$ - acceleration of gravity $\left(g=9,81 \mathrm{~m} / \mathrm{s}^{2}\right) ; b$ - width of the bridge.

Standard for water flow should be calculated by the derivative $V_{C}$, Formula (7).

$$
\sigma_{Q_{c}}=\sqrt{\left(1.15 \cdot \frac{\bar{V}_{C}^{1 / 3}}{g^{2 / 3}} \cdot b \cdot \sigma_{V c}\right)^{2}}
$$

It should be noted that the reliability of a road and its engineering facilities depends on a number of parameters described above. The authors have assessed the statistical characteristics and parameters of a road and engineering facilities derived from the statistical distributions [8-10]. The statistical data on the material properties, geometric dimensions of road elements and engineering structures, and their statistical distribution acted as the initial data.

On their basics the distribution of the analyzed parameters is made by using standardized force deformation relationship [11]. The authors prove this statement by the example providing the following results.

\section{Results}

According to statistical research, variation coefficient for soil density $(\gamma)$, angle of internal friction $(\varphi)$ and adhesion (c) is $V=15 \%$. The average values for $\gamma, \varphi$ and $c$ are: $\gamma=2000$ $\mathrm{kg} / \mathrm{m}^{3} ; \varphi=7,5 €$ and $c=0,006$.

Then the standards are found for $\gamma, \varphi, c$

$$
\begin{aligned}
& \sigma_{\gamma}=V_{\gamma} \cdot \bar{\gamma}=0.15 \cdot 2000=300 \mathrm{~kg} / \mathrm{m}^{3} \\
& \sigma_{\varphi}=V_{\varphi} \cdot \operatorname{tg} \bar{\varphi}=0.15 \cdot \operatorname{tg} 7.5=0,02 \\
& \sigma_{c}=V_{c} \cdot \bar{c}=0.15 \cdot 0.006=0.0009
\end{aligned}
$$

Now the standard gradient $\mathrm{m}$, average value and variation coefficient should be found. 


$$
\begin{aligned}
& \sigma_{m}=\sqrt{\left(\frac{1}{\operatorname{tg} \bar{\varphi}^{2}+\frac{\bar{c}}{\bar{p}_{h}}} \cdot \sigma_{\psi}\right)^{2}+\left(\frac{1}{\operatorname{tg} \bar{\varphi}+\frac{\bar{c}^{2}}{\bar{p}_{h}}} \cdot \sigma_{c}\right)^{2}+\left(\frac{1}{\operatorname{tg} \bar{\varphi}+\frac{\bar{c}}{\cos ^{2} \varphi \cdot \bar{\gamma}^{2}}} \cdot \sigma_{\gamma}\right)^{2}}= \\
& =\sqrt{\left(\frac{1}{\left(\operatorname{tg} 7.5^{\circ}\right)^{2}+\frac{0.006}{\cos ^{2} 7.5^{0} \cdot 6 \cdot 2000}} \cdot 300\right)^{2}+\left(\frac{1}{0.13+\frac{0.006^{2}}{0.98 \cdot 6 \cdot 2000}} \cdot 0.0009\right)^{2}+} \\
& \sqrt{\left(\frac{1}{0.13+\frac{0.006}{0.98 \cdot 6 \cdot 2000^{2}}} \cdot 0.02\right)^{2}}=\sqrt{16.66+0+0}=4.08 \\
& \bar{m}=\frac{1}{\operatorname{tg} \bar{\varphi}+\frac{\bar{c}}{\cos ^{2} \varphi \cdot h \cdot \bar{\gamma}}}=\frac{1}{0.13+\frac{0.006}{0.98 \cdot 6 \cdot 2000}}=7.69 \\
& v_{m}=\frac{\sigma_{m}}{\bar{m}}=\frac{4.08}{7.69}=0.53
\end{aligned}
$$

To determine the reliability of the slope the normative values for the slope should be used.

For loam the slightest slope is $1: 1.5$

$V$ is $10 \%$, thus:

$$
\sigma_{V}=V \cdot \bar{m}=1.5 \cdot 0.1=0.15
$$

The reliability is calculated by the Formula (3):

$$
P=f\left(\frac{7.69-1.5}{\sqrt{4.08^{2}+0.15^{2}}}\right)=f(1.51)=0.93
$$

The reliability of the superelevation can be calculated by the Formula (4) following the statement of the research that provides the best conditions for car passage along the curve when $\mu=0.05 h 0.1$. Then the value of $\mu$ and the average values of $\sigma_{\mu}$ and $v_{\mu}$ are calculated.

$$
\begin{gathered}
\bar{\mu}=\frac{0.1+0.05}{2}=0.075 \\
\sigma_{\mu}=\sqrt{\frac{(0.075-0.05)^{2}+(0.075-0.1)^{2}}{2-1}}=0.035 \\
\nu_{\mu}=\frac{0.035}{0.075}=0.47
\end{gathered}
$$

For speed $\mathrm{V}$ we take variation coefficient $v_{V} \cong_{0.2}$, while the average value is $\bar{V}=120$ $\mathrm{km} / \mathrm{h}$.

We get: 


$$
\sigma_{V}=\bar{V} \cdot v_{V}=1200.2=24 \mathrm{~km} / \mathrm{h}
$$

Then the standard for the superelevation gradient is calculated by the Formula (5).

$$
\begin{gathered}
\sigma_{i}=\sqrt{\left(2 \cdot \frac{33.33}{9.81 \cdot 800} \cdot 6.67\right)^{2}+0.035^{2}}=0.036=36 \% \\
\bar{i}=\frac{\bar{V}^{2}}{g \cdot R}-\bar{\mu}=\frac{(120 / 3.6)^{2}}{9.81 \cdot 800}-0.075=0.067=67 \% \\
v_{i}=\frac{\sigma_{i}}{\bar{i}}=\frac{0.036}{0.067}=0.53
\end{gathered}
$$

For Category II road with the turning radius from $2000 \mathrm{~m}$ to $1000 \mathrm{~m}$ the superelevation gradient is $200 \%$.

$V$ take $10 \%$, thus:

$$
\sigma_{V}=V \cdot \bar{i}=20 \cdot 0.1=2 \%
$$

Then the reliability is determined by the following:

$$
P=f(\mathrm{Z})=f\left(\frac{\bar{m}-m_{\text {norm }}}{\sqrt{\sigma_{m}^{2}+\sigma_{\text {norm }}^{2}}}\right)=f\left(\frac{67-20}{\sqrt{36^{2}+2^{2}}}\right)=f(1,3)=0.903
$$

Then the reliability of a bridge capability is calculated, Formula (6).

$$
Q_{C}=1.35\left(\frac{1.45 \cdot V_{C}}{g}\right)^{2 / 3} \cdot b=1.73 \cdot \frac{V_{C}^{2 / 3}}{g^{2 / 3}} \cdot b
$$

In the given formula only the flow velocity under the bridge is variable.

The average value of flow velocity, standard and its variation coefficient are the following.

$$
\begin{gathered}
\bar{V}_{C}=\frac{5+7}{2}=6 \mathrm{~m} / \mathrm{s} \\
\sigma_{\bar{V}_{C}}=\sqrt{\frac{(6-5)^{2}+(6-7)^{2}}{2-1}}=1.41 \mathrm{~m} / \mathrm{s} \\
v_{V_{C}}=\frac{1.41}{6}=0.23
\end{gathered}
$$

Standard for water flow is determined by the Formula (7).

$$
\begin{gathered}
\sigma_{Q_{C}}=\sqrt{\left(1.15 \cdot \frac{\bar{V}_{C}^{1 / 3}}{g^{2 / 3}} \cdot b \cdot \sigma_{V_{c}}\right)^{2}}=\sqrt{\left(1.15 \cdot \sqrt[3]{\frac{6}{9.81^{2}}} \cdot 10 \cdot 1.41\right)^{2}}=6.42 \mathrm{~m}^{3} / \mathrm{s} \\
\bar{Q}_{C}=1.73 \cdot\left(\frac{\bar{V}_{C}}{g}\right)^{2 / 3} \cdot b=1.73 \cdot\left(\frac{6}{9.81}\right)^{2 / 3} \cdot 10=12.51 \mathrm{~m}^{3} / \mathrm{s}
\end{gathered}
$$




$$
v_{V_{C}}=\frac{\sigma_{V_{c}}}{\bar{Q}_{C}}=\frac{6.42}{12.51}=0.51
$$

The distribution of statistical characteristics [12-15] should be used to determine the reliability of engineering facilities.

\section{Discussion}

To determine the failure of a bridge, you must subtract one distribution from another, that is $Q_{C}-Q_{L}$, Fig.1.

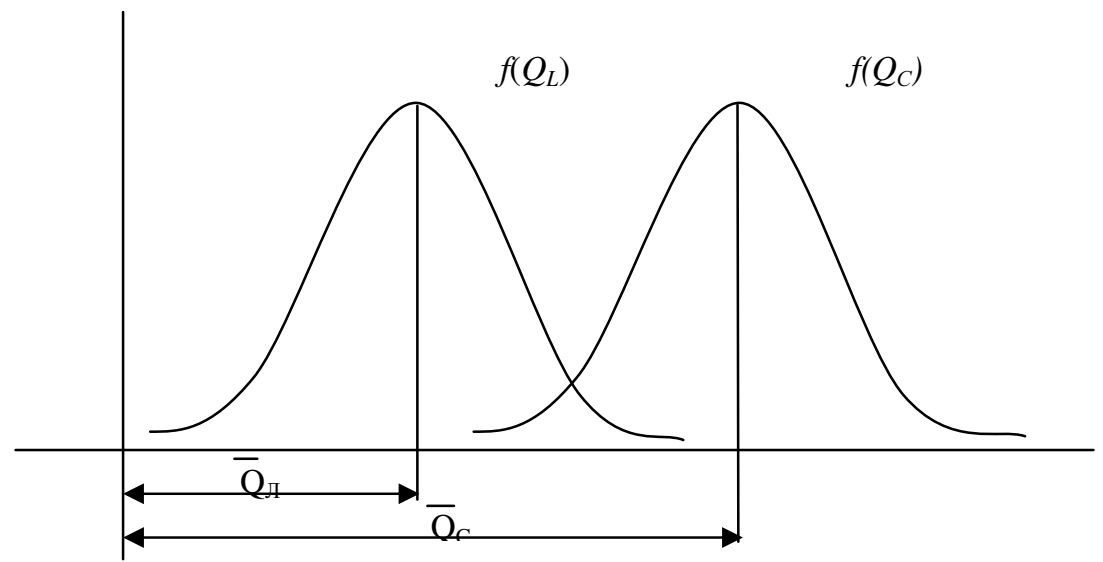

Fig. 1. Distribution of bridge failures.

The difference in these distributions will give the characteristic presence of zero on the abscissa axis, which divides the area below the distribution line into two parts, the risk is on the left while the reliability is on the right [16-17].

To determine zero it is necessary to find the value of $Z$.

At that we find $Z$ for $Q_{L}$ average and $\sigma_{\text {Л }}$ with probability value $\bar{a}_{\text {hour }} \quad 1,2,3$ и 4\%, (8), Fig. 2.

$$
Z=\frac{\bar{Q}_{C}-\bar{Q}_{L}}{\sqrt{{\sigma_{C}}^{2}+\sigma_{L}^{2}}}
$$

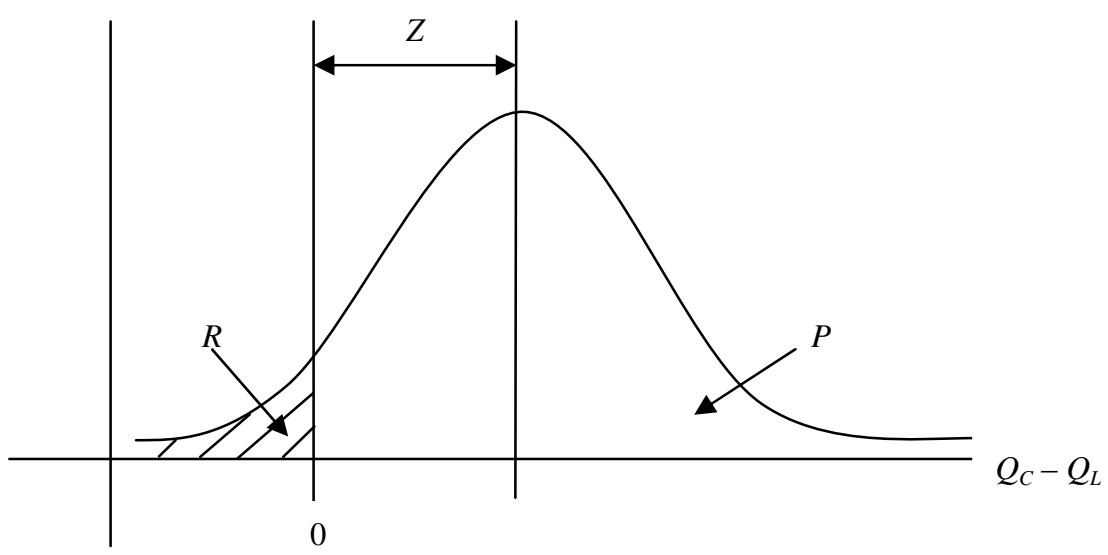

Fig. 2. Total bridge failure distribution. 
$4 \%$

$$
\begin{aligned}
& R=1-P=1-f(Z)=1-f\left(\frac{\bar{Q}_{C}-\bar{Q}_{L}}{\sqrt{\sigma_{C}{ }^{2}+\sigma_{L}{ }^{2}}}\right) \\
& R=1-f\left(\frac{12.51-7.87}{\sqrt{6.42^{2}+1,7^{2}}}\right)=1-f(0.697)=1-0.758=0.242
\end{aligned}
$$

$3 \%$

$$
R=1-f\left(\frac{12.51-8.64}{\sqrt{6.42^{2}+1.78^{2}}}\right)=1-f(0.581)=1-0.7257=0.2743
$$

$2 \%$

$$
R=1-f\left(\frac{12.51-9.57}{\sqrt{6.42^{2}+1, .9^{2}}}\right)=1-f(0.437)=1-0.6736=0.3264
$$

$1 \%$

$$
R=1-f\left(\frac{12.51-12.4}{\sqrt{6.42^{2}+3.07^{2}}}\right)=1-f(0.0155)=1-0.5=0.5
$$

The probability of exceeding $\bar{Q}_{L}$ и $\bar{a}_{\text {hour }}$ under the bridge for the Category II road is $1 \%$. The bridge failure should be 0.01 or $1 \%$, thus the reliability is 0.99 .

$$
\begin{gathered}
\left.\sigma_{Q_{C}}=\sqrt{\left(1.15 \cdot \frac{\bar{V}_{C}^{1 / 3}}{g^{2 / 3}} \cdot b \cdot \sigma_{V c}\right.}\right)^{2}=\sqrt{\left(1.15 \cdot \sqrt[3]{\frac{6}{9.81^{2}}} \cdot 16 \cdot 1.41\right)^{2}}=7.51 \mathrm{~m}^{3} / \mathrm{s} \\
\bar{Q}_{C}=1.73 \cdot\left(\frac{\bar{V}_{C}}{g}\right)^{2 / 3} \cdot b=1.73 \cdot\left(\frac{6}{9.81}\right)^{2 / 3} \cdot 16=18.32 \mathrm{~m}^{3} / \mathrm{s} \\
R=1-P=1-f(Z)=1-f\left(\frac{\bar{Q}_{C}-\bar{Q}_{L}}{\sqrt{\sigma_{C}{ }^{2}+\sigma_{L}^{2}}}\right) \\
R=1-f\left(\frac{18.32-12.4}{\sqrt{7.51^{2}+3.07^{2}}}\right)=1-f(2.35)=1-0.99=0.01
\end{gathered}
$$

It is evident that the specified bridge opening $b=10 \mathrm{~m}$ is not able to provide the passage of the storm drain $\bar{Q}_{L}$ with the necessary probability degree of failure even if the probability value $\bar{a}_{\text {hour }}$ is $4 \%$. Therefore for the given exceedance interval $\bar{a}_{\text {hour }}$ and $\bar{Q}_{L}$ it is necessary to calculate the required bridge length by using a pick method. It has been confirmed by the latest calculation studies.

\section{Conclusions}

The reliability of each of the components of roads and engineering structures on them is a great task. The reliability of the road is different depending upon the season. Thus it should 
be determined for spring-summer and autumn-winter periods. These estimates are independent. For solving it you should:

- know the factors that make up the parameters of roads and engineering facilities;

- make sequential and parallel connections;

- determine the statistical factors;

- evaluate the reliability of each factor.

The calculations got in the research make it possible to calculate the optimum length of a bridge opening with a specified reliability level of water runoff under the bridge.

\section{References}

1. L.G. Gordovskaya, Urban Construction and Architecture 1, 72-80 (2015) doi:10.17673/Vestnik.2015.01.12

2. A.N. Davydov, Tradition and Innovation in Building and Architecture, 162-165 (2015)

3. A.N. Davydov, Tradition and Innovation in Building and Architecture, 116-120 (2016).

4. T.V Dormidontova, Monograph, Sibak, 152 (2015)

5. T.V. Dormidontova, Internet journal Naukovedenie 2, 64-70 (2015)

6. T.V. Dormidontova, Patent to Useful Model 160415 (2016)

7. S.V. Evdokimov, Scientific Survey 22, 102-107 (2015)

8. B.G. Ivanov, Ways of Improving the Quality of Roads, 107-111 (2015)

9. B.G. Ivanov, Tradition and Innovation in Building and Architecture, 36-37 (2013)

10. B.G. Ivanov, Tradition and Innovation in Building and Architecture, 47 (2013)

11. E.I. Marinin, Urban Construction and Architecture 4, 80-84 (2014) doi:10.17673/Vestnik.2014.04.12

12. V.A. Pavlova, Ways of Improving the Quality of Roads, 158-168 (2015)

13. V.A. Pavlova, Tradition and Innovation in Building and Architecture, 156-161 (2015)

14. L.V. Pavlova, Urban Construction and Architecture 2, 96-103 (2016) doi:10.17673/Vestnik.2016.02.18

15. L.V. Pavlova, Ways of Improving the Quality of Roads, 168-176 (2015)

16. A.V. Filatova Ways of Improving the Quality of Roads, 206-211 (2015)

17. A.V. Filatova, Scientific Survey 7, 152-156 (2015) 